\title{
Processing emotional tone from speech in Parkinson's disease: A role for the basal ganglia
}

\author{
MARC D. PELL \\ McGill University, Montréal, Québec, Canada \\ and \\ CAROL L. LEONARD \\ Baycrest Centre for Geriatric Care, Toronto, Ontario, Canada
}

\begin{abstract}
In this study, individuals with Parkinson's disease were tested as a model for basal ganglia dysfunction to infer how these structures contribute to the processing of emotional speech tone (emotional prosody). Nondemented individuals with and without Parkinson's disease ( $n=21 /$ group) completed neuropsychological tests and tasks that required them to process the meaning of emotional prosody in various ways (discrimination, identification, emotional feature rating). Individuals with basal ganglia disease exhibited abnormally reduced sensitivity to the emotional significance of prosody in a range of contexts, a deficit that could not be attributed to changes in mood, emotional-symbolic processing, or estimated frontal lobe cognitive resource limitations in most conditions. On the basis of these and broader findings in the literature, it is argued that the basal ganglia provide a critical mechanism for reinforcing the behavioral significance of prosodic patterns and other temporal representations derived from cue sequences (Lieberman, 2000), facilitating cortical elaboration of these events.
\end{abstract}

There is accumulating evidence that the basal ganglia are functionally critical in the processing of meaning from voice tone, or speech prosody. Prosody refers to those variations in the pitch, duration (rhythm), and loudness of speech that inform listeners about the physiologicalstatus of their interlocutor and/or facets of their mental states, allowing for a number of important attributions to be drawn about the speaker that shape further aspects of the interpersonal event. Of key interest, speech prosody assumes a preeminent role in the cognitive mediation and recognition of stimuli referring to differentiated emotional states, such as happiness, anger, or fear, to name but a few (Scherer, 1993; Scherer, Banse, \& Wallbott, 2001).

The ability to recognize the emotive significance of prosodic cues in speech is believed to involve specialized mechanisms that engage distinct neural circuitry from more basic emotional processes and responses (Adolphs, 2002; Borod, 1993; Heilman, 1997) and from mechanisms that support analysis of the linguistic-propositional content of

This research was supported by a CIHR-IA New Investigator award granted to the first author (M.D.P.) and represents an Isabel Silverman Canada International Scientific Exchange Program project, supported by the Saul A. Silverman Family Foundation, Toronto, Canada (to C.L.L.). The assistance of Marta Fundamenski, Sarah AddlemanFrankel, Elmira Chan, Sam Hosseini, and Marlene Ecker in data collection and manuscript preparation is gratefully acknowledged. Correspondence concerning this article should be addressed to M. D. Pell, School of Communication Sciences and Disorders, McGill University, 1266 ouest, avenue des Pins, Montréal, PQ, H3G 1 A8 Canada (e-mail: marc.pell@mcgill.ca). prosody (Gandour, Wong, \& Hutchins, 1998; Pell, 1998; Pell \& Baum, 1997). There is steadily converging opinion that understanding emotional prosody activates a highly distributed neural architecture involving cortical and subcortical regions and interconnecting circuitry (Adolphs, Damasio, \& Tranel, 2002; Baum \& Pell, 1999; Kotz et al., 2003). Current descriptions of this circuitry, however, remain imprecise and are focused largely on defining the contributions of the right neocortex to this behavioral subsystem (Pell, 1998; Pihan, Altenmüller, \& Ackermann, 1997; Ross, Thompson, \& Yenkosky, 1997). Little attention has been directed toward elaborating the role of subcortical components of functional brain networks engaged in the decoding of emotional prosody. A survey of the small but growing literature on this topic yields prominent claims that the basal ganglia share the responsibility of processing emotion (and perhaps other meanings) from vocalprosodic cues; these ideas remain subject to rigorous, a priori evidence that informs the relationship between emotional prosody and basal ganglia functioning, the main objective of the present report.

Early evidence that the basal ganglia contribute to emotional prosody recognition has emerged, often post hoc, from neuropsychological group studies of patients with focal lesions of the basal ganglia; the subjects described in several reports have exhibited difficulties in discriminating or recognizing the emotional tone of prerecorded utterances, implicating the basal ganglia in these operations (Bradvik et al., 1991; Cancelliere \& Kertesz, 1990; Starkstein, Federoff, Price, Leiguarda, \& Robinson, 1994; Wed- 
dell, 1994). These conclusions intersect with recent functional neuroimaging studies of the healthy brain that have documented significant basal ganglia activation during the processing of vocal cues to emotion (Kotz et al., 2003; Morris, Scott, \& Dolan, 1999; but not Buchanan et al., 2000; George et al., 1996).

Further indications that the basal ganglia participate in emotional prosody decoding are garnered from behavioral investigations of adults with idiopathicParkinson's disease (PD). Onset of PD is marked by the interruption of dopaminergic neural projections from the substantia nigra pars compacta to the neostriatum, with progressive influences on other parts of the basal ganglia (ventral striatum) and the neocortex via thalamocortical circuits (Freeman et al., 2001). Although PD pathology is thus defined by the susceptibility of nerve cell populations and systems in several brain regions and is not a "pure" model of basal ganglia functioning, controlled examination of individuals in the early stages of PD is nonetheless a frequent and constructive approach by which to infer basal ganglia, particularly neostriatal, contributions to behavior and cognition. Indeed, several investigators have focused specifically on the comprehension of emotional prosody in nondemented individuals in early PD with mild to moderate motor signs; in the large majority of these reports, a significant association between basal ganglia compromise in PD and deficits in the recognition of emotion from prosody has been made (Blonder, Gur, \& Gur, 1989; Breitenstein, Daum, \& Ackermann, 1998; Breitenstein, Van Lancker, Daum, \& Waters, 2001; Lloyd, 1999; Pell, 1996; Scott, Caird, \& Williams, 1984; cf. Caekebeke, Jennekens-Schinkel, van der Linden, Buruma, \& Roos, 1991; Kan, Kawamura, Hasegawa, Mochizuki, \& Nakamura, 2002). Problems in decoding emotional prosody have also been attributed to basal ganglia degeneration in Huntington's disease (Speedie, Brake, Folstein, Bowers, \& Heilman, 1990; Sprengelmeyer et al., 1996). The accumulating work on PD and Huntington's disease converge with data from other clinical and neuroimaging sources that ascribe an important role for the basal ganglia in the processing of emotional tone from speech. This relationship merits comprehensive evaluation in order to advance ideas about what computational properties of the basal ganglia may be conducive to prosody decoding and about the relationship of this mechanism to cognitive-communicative functions already associated with the basal ganglia (Adolphs et al., 2002; Harrington, Haaland, \& Hermanowicz, 1998).

The present study was designed to extend knowledge of how the basal ganglia are implicated in the decoding of emotional information from prosody by comparing individuals with and without early PD on an array of tests sensitive to this form of information processing. Greater specificity about conditions that recruit basal ganglia mechanisms in the mediation of prosodic stimuli was achieved through direct comparison of prosodic abilities in three distinct tasks from which present conclusions in the literature are more selectively derived (involving discrimination, categorization, and continuousrating paradigms). Comprehen- sion of prosody was also compared with the processing of communicative symbols of emotion expressed in different sensory modalities (e.g., facial expressions) and in relation to cognitive alterations, such as reduced working memory capacity (Breitenstein et al., 2001), allowing the potential direct involvement of basal ganglia mechanisms in prosodic operations to be studied with greater certainty. The experimental design also allows commentary on the basal ganglia's role in the processing of certain "basic" emotions, such as disgust, addressing recent suggestions in this literature (Calder, Keane, Manes, Antoun, \& Young, 2000; Sprengelmeyer et al., 1996; Wang, Hoosain, Yang, Meng, \& Wang, 2003).

\section{METHOD}

\section{Subjects}

Twenty-one individuals with idiopathic PD without dementia, ranging in age from 51 to 83 years, were recruited to the study through a Self-Management Program at the Baycrest Centre for Geriatric Care in Toronto, Canada. Idiopathic PD was confirmed by a residing neurologist on the basis of accepted motor criteria (e.g., Calne, Snow, \& Lee, 1992). Participation in the study was restricted to individuals without other serious medical conditions (e.g., stroke, primary psychiatric disorder) or history of substance abuse. Absence of dementia in PD patients was verified by the research team at the onset of testing, using the Mattis Dementia Rating Scale. Motor disability of individuals within the PD group was in the mild severity range according to the Hoehn and Yahr and the unified PD rating scale (UPDRS) motor criteria; motor signs within the patient sample were characterized as left dominant $(n=11)$, right dominant $(n=7)$, or bilateral $(n=3)$. Except for 1 PD individual who chose to defer treatment, all the patients were optimally medicated during testing (on-state), distributed as follows: carbidopa/L-dopa $(n=17)$, d2agonist $(n=11)$, MAO-B inhibitor $(n=1)$, COMT inhibitor $(n=1)$, amantadine $(n=3)$, and anticholinergics $(n=1)$. One PD subject was also receiving an antidepressant (paroxetine). Patient subjects were matched on a one-to-one basis for sex, age, and educational status with 21 healthy aging controls (HC) living in the Montreal region. The control subjects exhibited a negative history of neurologic and psychiatric disease and were also screened for evidence of intact intellectual status on the Dementia Rating Scale. All the patient and control subjects passed a puretone audiometric screening to ensure acceptable hearing thresholds at frequencies important to speech intelligibility (minimum of $30 \mathrm{db}$ HL at $0.5,1,2$, and $4 \mathrm{kHz}$ ). Presence and severity of depression in all participants was estimated with the Hamilton Depression Inventory-Short Form (HI-SF). Table 1 summarizes the major demographic and clinical features of each subject group. Ethical approval of the study was granted by the McGill Faculty of Medicine Institutional Review Board and the joint Baycrest Centre/ University of Toronto ethics committee, and informed written consent was obtained from each subject prior to testing.

\section{Materials}

\section{Prosodic Stimuli}

All the stimuli used to tap aspects of prosodic processing in individuals with $\mathrm{PD}$ were recorded by four male and four female actors. Digital recordings of short nonsense utterances (e.g., Someone migged the pazing) and semantically biasing, well-formed utterances of comparable length (e.g., I didn't make the team) were elicited from each actor in five distinct emotional tones (happiness, pleasant surprise, anger, disgust, and sadness). Emotion identities were chosen for the purported "universality" of each underlying emotion, coupled with the desire to balance positive- and negative- valenced emo- 
Table 1

Demographic and Clinical Features of Subjects With Parkinson's Disease (PD) and Healthy Controls (HC)

\begin{tabular}{|c|c|c|c|c|}
\hline \multirow[b]{3}{*}{ Variable } & \multicolumn{4}{|c|}{ Group } \\
\hline & \multicolumn{2}{|c|}{$\mathrm{PD}^{*}$} & \multicolumn{2}{|c|}{$\mathrm{HC}^{*}$} \\
\hline & $M$ & $S D$ & $M$ & $S D$ \\
\hline Age (years) & 61.7 & 8.6 & 61.9 & 8.5 \\
\hline Education (years) & 16.0 & 3.7 & 16.0 & 2.6 \\
\hline Disease duration (years) & 3.9 & 1.9 & - & - \\
\hline Hoehn and Yahr rating score & 2.0 & 0.5 & - & - \\
\hline Motor UPDRS & 14.5 & 7.1 & - & - \\
\hline Mattis Dementia Rating Scale ${ }^{\dagger}$ & 141.2 & 2.1 & 142.5 & 1.8 \\
\hline Hamilton Depression Inventory & 4.7 & 3.4 & 2.3 & 2.9 \\
\hline
\end{tabular}

*Each group was composed of 10 female and 11 male subjects. †Total score, maximum $=144 . \quad$ Short form, maximum $=33$, increased scores indicate greater impairment.

tion targets within the dataset to the greatest extent possible. Emotionally inflected but semantically meaningless utterances were of paramount interest here because such stimuli effectively isolate cues of potential emotional significance to the prosodic channel (Pell \& Baum, 1997; Scherer et al., 2001). An extensive perceptual validation study of the prosodic stimuli was conducted to yield clear indications about the representational value of the recorded utterances to a group of young healthy listeners (see Pell, 2002, for details). On the basis of perceptual indicators, unambiguous items that achieved a minimum of $70 \%$ consensus about the emotion conveyed by the prosody (where chance recognition was 14\%) were used to construct the experimental tasks.

\section{Tests of Emotional Prosody Comprehension}

Prosodic stimuli were presented in three distinct conditions that varied how listeners processed and judged the underlying emotional significance of the stimulus. A common set of base stimuli, involving an equal number of exemplars produced by female and male speakers, contributed to the tasks within each condition.

Discrimination of emotional prosody. The ability to discriminate nonsense utterance pairs according to the emotional value of the prosody was assessed by eliciting a same/different judgment to 30 distinct trials (representing an equal ratio of same and different emotional pairings). The utterances included in each trial were presented serially separated by a 1 -sec interstimulus interval and always involved speakers of the same sex. The set of same trials reflected three distinct combinations of exemplars for each of the five emotions, whereas different trials were constructed by randomly combining exemplars of each emotional category with those for every other category at least once. The accuracy of the sameldifferent judgment was recorded.

Identification of emotional prosody. The ability to categorize the meaning conveyed by the prosodic form of the utterances was evaluated in three tasks in order to achieve a more sensitive analysis of abilities related to this ubiquitous measure of emotional prosody comprehension. Experimental task manipulations within this condition addressed whether (1) identifying the meaning of emotional prosody from nonsense utterances (i.e., pure prosody stimuli) was dependent on the number of emotional contrasts and corresponding response alternatives available to the listener and (2) identifying emotional prosody was more reliable when accompanied by redundant verbal-semantic cues in speech. Task comparisons would illuminate whether the off-line selectional demands associated with emotional processing and/or the need to weigh decisions on prosodic information in the speech signal alone would influence conclusions about basal ganglia contributions to the decoding of emotional prosody. A multiple-choice format with a closed set of verbal labels as response alternatives was employed in each task.

Pure prosody, three-choice (PP-3). The subjects listened to a series of nonsense stimuli intoned in one of three highly distinctive emotional tones (happiness, anger, or sadness). After listening to each utterance, the subjects identified which of the three emotions best corresponded to how the speaker was feeling $(8$ items $\times 3$ emotions $=24$ trials).

Pure prosody, five-choice ( $P P-5)$. The subjects listened to an expanded series of nonsense utterances representing five distinct emotional meanings (happiness, pleasant surprise, anger, disgust, and sadness). Following each trial, the subjects indicated which of the five emotions best corresponded with how the speaker was feeling ( 8 items $\times 5$ emotions $=40$ trials $)$.

Prosody + semantics, five-choice (PS-5). The subjects were presented well-formed utterances containing prosodic and verbalsemantic information that simultaneously biased one of five emotional meanings (happiness, pleasant surprise, anger, disgust, or sadness). For each trial, the subjects decided which label corresponded to how the speaker was feeling ( 8 items $\times 5$ emotions $=40$ trials $)$.

Emotional prosody feature rating. Comprehension of emotional prosody was further evaluated by requiring the subjects to monitor the prosodic form of the utterances for a predetermined emotional feature (e.g., happiness) and to rate the degree to which the selected attribute was present in the signal along a continuous scale (Adolphs \& Tranel, 1999). A single experiment was constructed involving the 40 nonsense utterances presented in the PP-5 identification task (8 items $\times 5$ emotions), plus 12 prosodically ambiguous nonsense stimuli known to represent a blend of emotional attributes based on the perceptual data for these items. The subjects listened to a randomized list of the 52 nonsense utterances on five separate occasions. On each occasion, the subjects were instructed to attend to only one of the five target emotions and to rate each stimulus for "how much of the emotion was being expressed," on a scale from 0 (not at all) to 5 (very much). This process yielded five separate prosodic ratings for each of the 52 stimuli, providing an index of how each subject detected intended emotional features of each item, relative to other possible attributes of the stimulus, and of each subject's global sensitivity to the set of five possible meanings that may be partially encoded by prosodic stimuli. At the same time, this approach mitigates off-line selectional demands associated with categorizing stimuli in reference to a single emotion.

\section{Neuropsychological Background Testing}

The testing battery included specific tasks designed to provide a broader context of prosodic- and emotional-processing abilities in individuals with basal ganglia compromise, as well as standardized tasks of cognitive functioning in several areas critical to data interpretation.

Control tasks of emotional and prosodic processing. Identification of emotion to verbal descriptions. Each subject listened to 10 sentences describing prototypical situations that might elicit one of the five target emotions ( 2 items $\times 5$ emotions). The subjects indi- 
cated how they would feel in each situation from the set of five emotional labels used in prosody tasks.

Identification of emotion from facial expressions. The subjects were presented a series of 40 static photographs of facial expressions ( 8 items $\times 5$ emotions) posed by the same actors as those who had recorded the prosodic stimuli (Pell, 2002). The subjects identified from a set of verbal labels which of the five emotions represented how the actor was feeling.

Discrimination of nonemotional auditory information. The ability to process segmental and prosodic cues in nonemotional contexts was evaluated in two discrimination tasks in which a same/different judgment was rendered: the Benton Phoneme Discrimination Subtest (for segmental information) and a task in which the subjects discriminated 24 nonsense utterance pairs according to the intrasentential location of emphatic stress (for nonemotional prosodic information). The stimuli in the latter task contained sentenceinitial, sentence-final, or no emphatic stress and were recorded by the same speakers as those who had prepared the emotional stimuli. An equal number of same and different emphasis trials were randomly presented.

Standardized neuropsychological tests. Neuropsychological functions that support prosodic-communicative processes were evaluated in an attempt to dissociate contributions of the basal ganglia to emotional prosody from alterations in more basic cognitive domains susceptible to these pathophysiological changes in early PD. Execution of prosody comprehension tasks required the subjects to hold auditory information in memory during processing and, while generating a response, to visually attend to verbal labels or other response prompts and to shift mental sets across tasks that required different types of processing and responses to highly similar auditory materials. Tests administered were the Forward Digit Span, a measure of verbal working memory span (Tompkins, Bloise, Timko, \& Baumgaertner, 1994), the Trail-Making Test, and the Wisconsin Card Sorting Test (WCST). The Attention subtest of the Mattis Dementia Rating Scale was also examined separately for each subject in order to elaborate information on basic attentional functioning.

\section{Procedure}

The subjects were tested independently in a quiet laboratory environment, at a time of day at which motor symptoms were typically least severe in the case of the PD subjects. Testing always began with the dementia and audiometric screenings, followed by administration of emotional prosody and neuropsychological tests intermixed in a quasirandom order over four testing sessions of approximately $1 \mathrm{~h}$ each. Tasks within each emotional prosody condition were separated whenever possible and were assigned to different sessions in order to minimize stimulus repetition across tasks during a single session and to limit possible fatigue or complacency induced by extended involvement in a particular task. The four sessions were fully randomized for presentation order within the PD group and then were matched with controls, and each session was separated by an interval of at least 1 week to mitigate familiarity with prosodic stimuli that were sometimes repeated across conditions. Presentation of emotional stimuli within prosody tasks was automatically randomized by Superlab software (Cedrus Corporation) and was played over high-quality volume-adjustable headphones. The subjects were free to indicate a response verbally or by pointing to their decision on the computer screen in front of them, without time limitations. All decisions were recorded by the examiner. Prosody and control tasks were preceded by a verbal explanation of the task requirements and a brief practice block. The subjects were paid a nominal fee at the end of testing to cover travel-related expenses and inconvenience associated with their participation.

\section{RESULTS}

The ability of PD patients to detect the meaning of emotional prosody in the discrimination, identification, and feature-rating conditions was examined separately and then compared across conditions and in relation to background/neuropsychological variables. Table 2 highlights

Table 2

Comprehension of Emotional Prosody by Condition for the Parkinson's Disease (PD) and Healthy Control (HC) Groups

\begin{tabular}{|c|c|c|c|c|c|}
\hline \multirow[b]{3}{*}{ Condition } & \multirow[b]{3}{*}{ Measure } & \multicolumn{4}{|c|}{ Group } \\
\hline & & \multicolumn{2}{|c|}{$\mathrm{PD}$} & \multicolumn{2}{|c|}{$\mathrm{HC}$} \\
\hline & & $M$ & $S D$ & $M$ & $S D$ \\
\hline Discrimination & Total correct ( /30) & 21.8 & 3.4 & 23.6 & 2.5 \\
\hline \multicolumn{6}{|l|}{ Identification } \\
\hline PP-3 & Total correct (/24) & 20.6 & 2.5 & 22.9 & 2.2 \\
\hline PP-5 & Total correct $(/ 40)$ & 27.4 & 4.9 & 31.6 & 3.4 \\
\hline PS-5 & Total correct (/40) & 33.0 & 3.6 & 35.1 & 3.2 \\
\hline \multicolumn{6}{|l|}{ Emotional feature rating } \\
\hline \multirow[t]{3}{*}{ Target $=$ happiness } & Mean target feature rating (scale $0-5$ ) & 2.93 & 1.00 & 3.11 & 0.75 \\
\hline & Proportion of " $4+5$ " target ratings & 0.39 & 0.29 & 0.42 & 0.30 \\
\hline & Overall emotional sensitivity* & 0.62 & 0.36 & - & - \\
\hline \multirow[t]{3}{*}{ Target $=$ pleasant surprise } & Mean target feature rating (scale, $0-5$ ) & 3.26 & 0.95 & 3.43 & 0.83 \\
\hline & Proportion of " $4+5$ " target ratings & 0.45 & 0.31 & 0.57 & 0.29 \\
\hline & Overall emotional sensitivity & 0.65 & 0.31 & - & - \\
\hline \multirow[t]{3}{*}{ Target $=$ anger } & Mean target feature rating (scale $0-5$ ) & 3.27 & 1.10 & 3.62 & 0.76 \\
\hline & Proportion of " $4+5$ " target ratings & 0.50 & 0.32 & 0.60 & 0.33 \\
\hline & Overall emotional sensitivity & 0.58 & 0.23 & - & - \\
\hline \multirow[t]{3}{*}{ Target $=$ disgust } & Mean target feature rating (scale, $0-5$ ) & 3.53 & 0.83 & 3.86 & 0.69 \\
\hline & Proportion of " $4+5$ " target ratings & 0.62 & 0.30 & 0.69 & 0.27 \\
\hline & Overall emotional sensitivity & 0.54 & 0.14 & - & - \\
\hline \multirow[t]{3}{*}{ Target $=$ sadness } & Mean target feature rating (scale, $0-5$ ) & 3.21 & 1.12 & 3.75 & 0.97 \\
\hline & Proportion of " $4+5$ " target ratings & 0.49 & 0.33 & 0.65 & 0.33 \\
\hline & Overall emotional sensitivity & 0.68 & 0.24 & - & - \\
\hline
\end{tabular}

* Represents the correlation of the set of five emotional ratings each PD subject assigned to target stimuli, in comparison with the HC group mean (see Adolphs \& Tranel, 1999). 
key measures of group performance in each condition of emotional prosody decoding.

\section{Emotional Prosody Decoding Within and Across Conditions}

Discrimination. The ability of listeners to accurately judge whether the emotional value of prosodic cues in paired nonsense utterances was the same or different was less reliable overall in the PD $(73 \% \pm 11 \%)$ than in the $\mathrm{HC}(79 \% \pm 8 \%)$ group. This difference represented a strong trend in the data that was only marginally significant $[t(40)=-1.91, p=.06]$. Further analysis of discrimination abilities briefly probed whether individuals in the two groups were equally sensitive to underlying contrasts in the emotional value of prosodic patterns for same versus different trials, following a signal detection analysis approach. The proportion of different emotion trials that each subject discriminated correctly (hit) and the proportion of same trials that subjects incorrectly judged to be distinct (false alarm) were computed for each of the 42 subjects. Mean hit rates were significantly reduced in the PD group (.67 \pm .19$)$, as compared with the HC group $(.85 \pm .1)$, overall $[t(40)=-3.78, p<.001]$, implying reduced sensitivity within the PD group to actual differences in the communicative significance of prosodic cues present in speech. Mean rates for false alarms were less disparate between groups and did not vary significantly $[\mathrm{PD}=.22 \pm .17, \mathrm{HC}=.28 \pm .15 ; t(40)=-1.18, p>.24]$.

Identification. The ability to explicitly categorize the meaning of emotional prosody from a set of verbal labels was captured by the proportion of correct responses in each of three distinct identification tasks (PP-3, PP-5, and PS-5). Separate group $\times$ emotion repeated measures analyses of variance (ANOVAs) involving two levels of group
(PD or HC) and three or five levels of emotion (PP-3, happiness, anger, or sadness; PP-5 and PS-5, happiness, pleasant surprise, anger, disgust, or sadness) were run on data representing each task. The results established that emotional prosody recognition was significantly inferior in the PD than in the HC group in the PP-3 and PP-5 tasks, each of which required subjects to derive their interpretations strictly from prosodic components of the signal [group main effect: $F_{\mathrm{PP}-3}(1,40)=10.53, p=.002 ; F_{\mathrm{PP}-5}(1,40)=$ $10.54, p=.002]$. Group differences were highly robust irrespective of the number of stimulus-response distinctions associated with the task (three or five), a variable that has been poorly controlled in the broader literature involving this paradigm.

Patterns described for pure prosody tasks (PP-3, PP-5) contrast with how the subjects identified emotional prosody from speech that encoded prosodic and verbal-semantic indicators of emotion (PS-5), where the accuracy of the PD subjects was reduced, but not reliably, from that of the HC subjects [group main effect $=$ n.s., $F(1,40)=3.62$, $p=.06]$. Figure 1 summarizes the impact of basal ganglia compromise on emotional prosody recognition as an index of the number of response alternatives and the types of cues available across identification tasks. It is noteworthy that the emotion variable exerted a significant but independent influence on prosody recognition rates in each of the three identification tasks $\left[F_{\mathrm{PP}-3}(2,80)=6.72, p=\right.$ $.007 ; F_{\mathrm{PP}-5}(4,160)=10.61, p<.001 ; F_{\mathrm{PS}-5}(4,160)=$ $14.06, p<.001]$; the precise relationship among emotion categories, as reflected in their relative identification scores and the types of confusions made, was complex and varied according to the specific task manipulations (Pell, 2002; Scherer et al., 2001). More critical here, there was no evidence that the groups were differentially sensi-

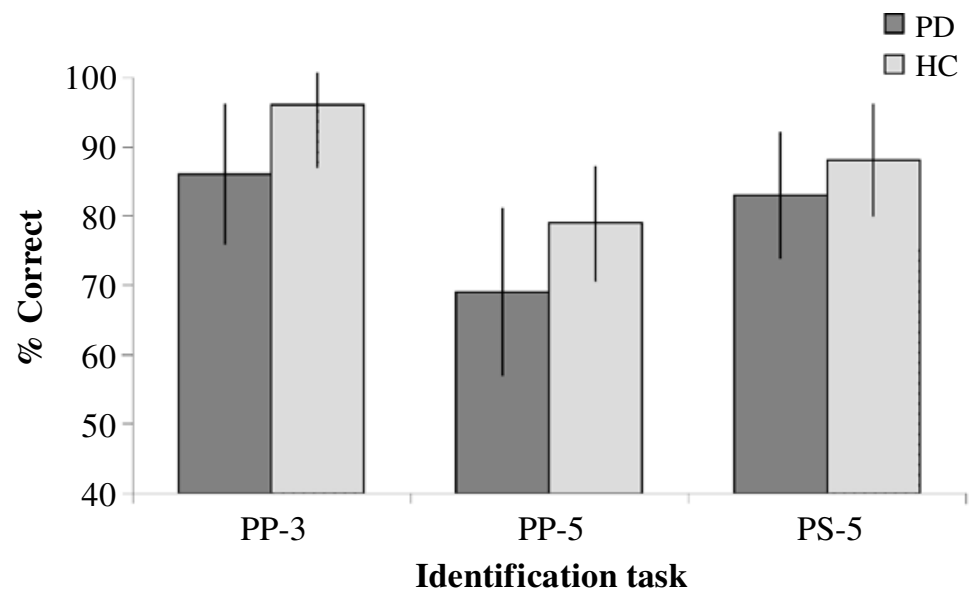

Figure 1. Identification of emotional prosody by Parkinson's disease (PD) and healthy control (HC) subjects as a function of available cues and task response parameters. Prosodic interpretations were based solely on prosodic features of utterances in a three-choice (PP-3) or five-choice (PP-5) paradigm or on congruent prosodic and verbal-semantic features of similar utterances in a five-choice paradigm (PS-5). 
Table 3

Distribution of Group Responses to Five Target Emotions in the Identification Condition, by Task

Distribution of Responses (\%)

\begin{tabular}{|c|c|c|c|c|c|c|c|c|c|c|c|}
\hline \multirow[b]{3}{*}{ Target Emotion } & & & & & & & & & & & \\
\hline & \multicolumn{6}{|c|}{ PD Group } & \multicolumn{5}{|c|}{ HC Group } \\
\hline & Task & Happiness & Surprise & Anger & Disgust & Sadness & Happiness & Surprise & Anger & Disgust & Sadness \\
\hline \multirow[t]{3}{*}{ Happiness } & PP-3 & 75 & - & 13 & - & 12 & 93 & - & 5 & - & 2 \\
\hline & PP-5 & 66 & 23 & 4 & 4 & 3 & 80 & 18 & 1 & 0 & 1 \\
\hline & PS-5 & 89 & 6 & 0 & 0 & 5 & 96 & 4 & 0 & 0 & 0 \\
\hline \multirow[t]{3}{*}{ Pleasant surprise } & PP-3 & - & - & - & - & - & - & - & - & - & - \\
\hline & PP-5 & 34 & 61 & 2 & 2 & 1 & 37 & 60 & 1 & 2 & 0 \\
\hline & PS-5 & 27 & 72 & 0 & 0 & 1 & 32 & 68 & 0 & 0 & 0 \\
\hline \multirow[t]{3}{*}{ Anger } & PP-3 & 6 & - & 90 & - & 4 & 1 & - & 98 & - & 1 \\
\hline & PP-5 & 8 & 10 & 63 & 17 & 2 & 2 & 6 & 75 & 17 & 0 \\
\hline & PS-5 & 1 & 6 & 80 & 12 & 1 & 0 & 2 & 85 & 13 & 0 \\
\hline \multirow[t]{3}{*}{ Disgust } & PP-3 & - & - & - & - & - & - & - & - & - & - \\
\hline & PP-5 & 2 & 11 & 15 & 67 & 5 & 2 & 8 & 11 & 76 & 3 \\
\hline & PS-5 & 0 & 4 & 7 & 83 & 6 & 0 & 4 & 2 & 92 & 2 \\
\hline \multirow[t]{3}{*}{ Sadness } & PP-3 & 3 & - & 5 & - & 92 & 2 & - & 2 & - & 96 \\
\hline & PP-5 & 6 & 5 & 5 & 7 & 77 & 0 & 3 & 0 & 2 & 95 \\
\hline & PS-5 & 1 & 1 & 8 & 1 & 89 & 0 & 0 & 0 & 2 & 98 \\
\hline
\end{tabular}

Note-The distribution of responses was based on 168 items per target emotion; the cells in boldface present identification rates for intended target emotions. PD, Parkinson's disease; HC, healthy controls; PP-3, pure prosody (prosodic cues only), three-choice task; PP-5, pure prosody, five-choice task; PS-5, prosody + semantic cues, five-choice task.

tive to these relationships in any of the three tasks [all group $\times$ emotion interactions $=$ n.s., $F_{\mathrm{PP}-3}(2,80)=2.48$, $p=.11 ; F_{\mathrm{PP}-5}(4,160)=1.21, p=.31 ; F_{\mathrm{PS}-5}(4,160)=0.89$, $p=.47]$. Table 3 demonstrates the full distribution of responses obtained for each target emotion by group according to differences in the availability of emotion cues and/or response requirements of the PP-3, PP-5, and PS5 tasks.

Emotional feature rating. The ability of the subjects to detect and rate specific emotional qualities of prosodic stimuli apart from the need to explicitly categorize this information verbally was first evaluated by analyzing how the subjects judged intended emotional targets of the prosodic stimuli. The subset of ratings reflecting how "happy" a speaker sounded to the subjects when the actual target of the item was happiness was determined, and so forth for each emotion, allowing inferences about "how much" of each target emotion was correctly detected. A high frequency of ratings at the upper end of the ordinal scale was expected, owing to rigorous preselection of the prosodic stimuli according to their perceptual emotion identity.

The absolute frequency distribution of responses obtained at each interval of the 6-point rating scale was computed separately by group and emotion $(8$ items $\times 5$ emotions $\times 21$ subjects $=840$ data points per group), and a series of chi-square tests was performed to determine the independence of the group frequency distributions pertaining to each emotion. Group membership was a significant factor in the ratings given to stimuli portraying disgust $\left[\chi^{2}(5)=12.65, p<.05\right]$ and sadness $\left[\chi^{2}(5)=\right.$ $11.52, p<.05]$, but not to stimuli conveying happiness, pleasant surprise, or anger (all remaining $p \mathrm{~s}>.10$ ). Analysis of the group rating distributions summed across emotions (involving 840 observations per group) indi- cated that recognition of target emotions from prosody was significantly influenced by the presence of PD overall $\left[\chi^{2}(5)=21.51, p<.001\right]$. As is demonstrated in Figure $2 \mathrm{~A}$, group differences in prosody ratings were largely explained by an attenuation in the perceived presence of target emotions in the stimuli by individuals with PD, evidenced by a systematic downward shift in responses obtained at the upper end of the 6-point continuum. There was a $16 \%$ decline in the number of prosodic stimuli individuals with PD rated as containing very much of the target emotions (i.e., a " 4 " or " 5 " response), coupled with a $37 \%$ increase in not at all (i.e., "0" or " 1 ") responses to the same stimuli by the PD relative to the $\mathrm{HC}$ group. These data strongly indicate that PD patients were less adept than healthy listeners at recognizing prominent emotional features of prosodic stimuli overall, with the possibility of more selective difficulties in the recognition of disgust and sadness.

The global sensitivity of listeners to the five emotional qualities potentially associated with each prosodic stimulus was examined briefly according to a prominent approach in this literature (see Adolphs \& Tranel, 1999, and Adolphs, Tranel, Damasio, \& Damasio, 1995, for details and a rationale). The set of five emotional ratings that each PD subject assigned to a single prosodic stimulus was correlated with the set of mean ratings given to that item by the $21 \mathrm{HC}$ subjects as a whole, and the correlations were transformed and arithmetically averaged across items expressing the same emotion. As is portrayed in Figure $2 \mathrm{~B}$, correlations between the $\mathrm{PD}$ and the $\mathrm{HC}$ groups for individual emotions ranged from a low of .54 for disgust to a high of .68 for sadness (anger $=.58$, happiness $=$ .62 , pleasant surprise $=.65$ ), with a mean similarity rating of .61 in the rating condition overall. Correlations fur- 


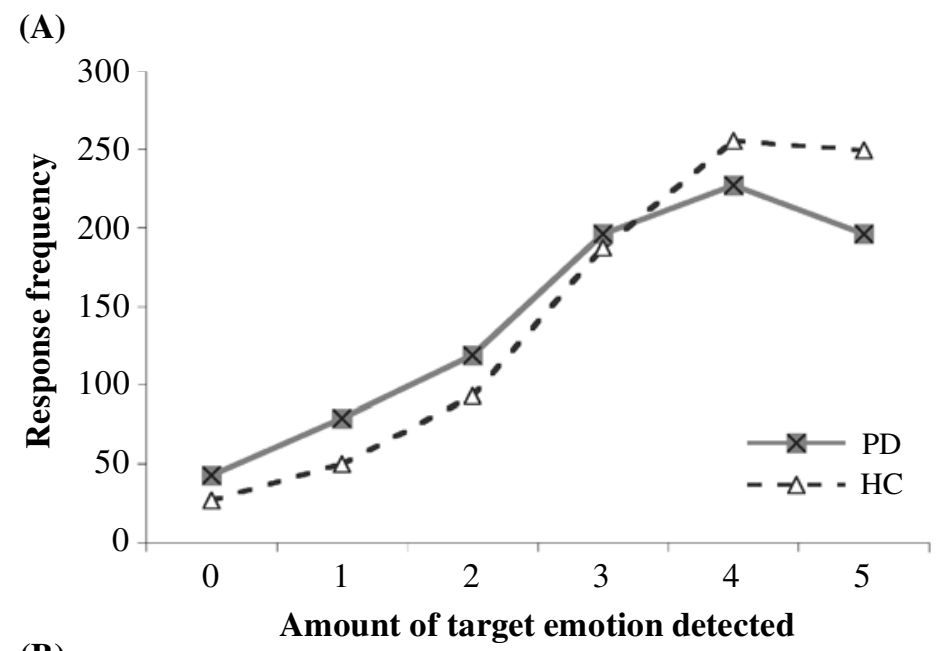

(B)

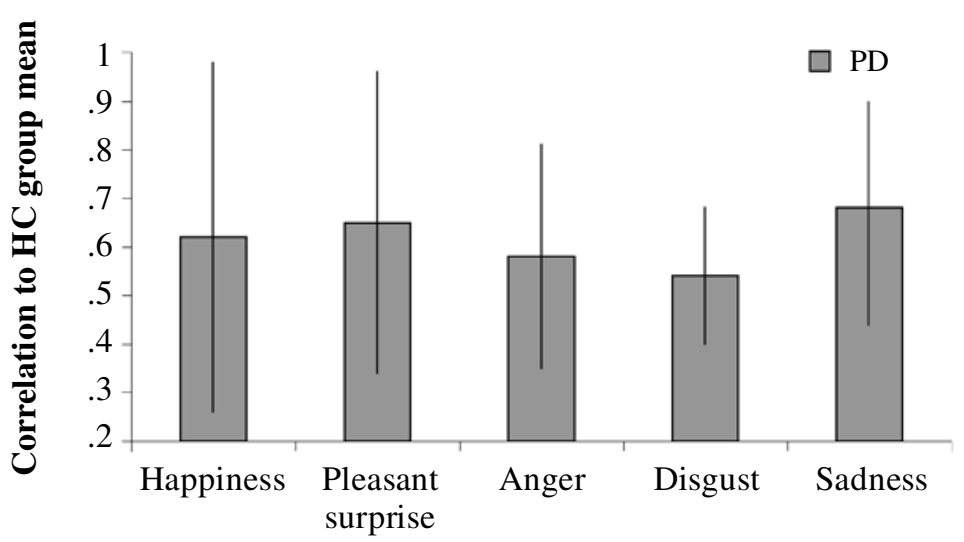

\section{Target emotion}

Figure 2. Sensitivity of Parkinson's disease (PD) subjects to prosody in the emotional-feature-rating condition: (A) Overall group frequency of responses observed when emotional prosody was rated for its intended target quality along a 6-point scale of increasing presence of the target emotion (collapsed across emotions). (B) Mean correlation of the set of five emotional ratings each PD subject assigned to prosodic stimuli, as was compared with the $\mathrm{HC}$ group mean, separated by intended target emotion.

ther from 1 have been viewed in light of increased impairment from normal patterns of sensitivity to a range of prosodic features (e.g., Adolphs et al., 1995), although it is noteworthy that for the present data the distance of the PD group's overall sensitivity ratings did not vary significantly as a function of the emotional target of the stimulus (one-way repeated measures ANOVA on the mean $Z$ transformed correlation coefficients for the five emotions, $p=.27$ ). Previous norms for these stimuli were lacking, precluding a direct test of disease status on sensitivity ratings according to this approach.

Emotional prosody decoding across conditions. An additional analysis compared the emotional prosody decoding skills of individuals in the PD and the HC groups in a direct manner across the three conditions. This analysis focused on dependent measures reflecting how the subjects derived meaning from prosodic cues alone (i.e., from nonsense stimuli) in tasks involving all five emotional distinctions (discrimination, PP-5, emotion feature rating). Discrimination and identification (PP-5) abilities were represented by the proportion of correct responses in each condition, whereas accuracy in the rating condition was represented by the proportion of combined " 4 " and " 5 " ratings that each subject gave to intended target emotions when judging that feature (out of 40 possible tokens). There was a strong overall relationship in the subjects' ability to derive the emotional significance of prosody for purposes of discrimination and identification $(r=.53$, $p<.05$ ), both of which were unrelated to how well the listeners detected target emotion features in the rating con- 
Table 4

Neuropsychological Performance Measures for the Parkinson's Disease (PD) and Healthy Control (HC) Groups

\begin{tabular}{|c|c|c|c|c|}
\hline \multirow[b]{3}{*}{ Variable } & \multicolumn{4}{|c|}{ Group } \\
\hline & \multicolumn{2}{|c|}{$\mathrm{PD}$} & \multicolumn{2}{|c|}{$\mathrm{HC}$} \\
\hline & $M$ & $S D$ & $M$ & $S D$ \\
\hline \multicolumn{5}{|c|}{ Emotion/Prosody Control Tasks } \\
\hline Emotion identification to verbal description (/10) & 7.7 & 1.7 & 8.1 & 1.4 \\
\hline Emotion identification from faces*(/40) & 35.5 & 3.2 & 36.1 & 3.1 \\
\hline Benton phoneme discrimination $(/ 30)$ & 26.4 & 3.2 & 28.3 & 1.7 \\
\hline Discrimination of nonemotional prosody $(/ 24)$ & 19.6 & 2.4 & 21.6 & 1.2 \\
\hline \multicolumn{5}{|c|}{ Tests of Cognitive/Executive Resources } \\
\hline Dementia Rating Scale, Attention subtest (/37) & 36.5 & 0.8 & 36.8 & 0.4 \\
\hline Auditory digit span, forward & 7.3 & 1.0 & 7.2 & 1.0 \\
\hline Auditory working memory/listening span (/42) & 36.2 & 3.6 & 39.7 & 2.3 \\
\hline Trail-Making Test B (seconds to complete) & 103.4 & 48.5 & 87.7 & 17.8 \\
\hline \multicolumn{5}{|l|}{ Wisconsin Card Sorting Test } \\
\hline Categories $($ maximum $=6)$ & 4.6 & 2.1 & 5.3 & 1.5 \\
\hline Nonperseverative errors $(\%)$ & 11.3 & 7.6 & 9.9 & 4.5 \\
\hline Perseverative errors $(\%)$ & 17.4 & 15.0 & 11.9 & 7.7 \\
\hline
\end{tabular}

dition (both $p \mathrm{~s}>.20)$. A group (PD or HC) $\times$ condition (discrimination, identification, or rating) mixed-design ANOVA performed on these data revealed that PD status had a significant negative impact on the processing of emotional prosody across the three conditions [group: $F(1,40)=7.16, p=.01]$. The measure of decoding accuracy used to represent rating performance was significantly lower than accuracy in the other two conditions [condition: $F(2,80)=24.63, p<.001]$, but effects of condition did not vary between groups $(p=.78)$. A $2 \times 2 \times 5$ ANOVA that tested whether the groups differed across conditions in the recognition of specific target emotions (omitting discrimination data that did not reflect differences by emotion) reconfirmed the separate contributions of group and condition to the understanding of emotional prosody recognition, with no indications that group scores varied significantly according to emotional differences in the stimuli overall (two- and three-way interactions involving group and emotion, $F_{\mathrm{s}}<0.93, p \mathrm{~s}>.45$ ). Cursory inspection of PD patients with left- versus right-lateralized motor signs revealed no obvious differences in overall accuracy scores for prosody discrimination (left $=.75$, right $=.74)$, PP-5 identification (left $=.73$, right $=.68$ ), or emotional target rating $($ left $=.49$, right $=.49)$.

\section{Influence of Background Variables on Prosody Comprehension}

The PD and the HC groups were matched closely for demographic variables of sex, age, and years of formal education and did not differ according to these characteristics (review Table 1). All PD individuals showed high performance on the Mattis Dementia Rating Scale (all Dementia Rating Scale full scores $>137 / 144$, cutoff $=123$ ), although the overall PD group distribution was significantly lower on this measure $[t(40)=-2.15, p=.04]$. Additional neuropsychological performance measures obtained for the two groups are supplied in Table 4. A series of $t$ tests indicated that the PD and the HC groups performed comparably on measures of attention [Dementia Rating Scale attention subscore, $t(40)=-1.51, p=.14]$, simple auditory memory [forward digit span, $t(40)=0.15, p=.88$ ], and many "traditional" measures of frontal lobe executive functioning [Trails-B, time to complete, $t(40)=-1.28$, $p=.21$; WCST, categories achieved, $t(40)=-1.67, p=$ .10 ; perseverative errors, $t(40)=1.50, p=.14$; nonperseverative errors, $t(40)=0.71, p=.48]$. Diagnosis of PD was associated with elevated depression scores [HDI-SF: $t(40)=2.45, p=.02$ ], although only 3 subjects-2 PD and $1 \mathrm{HC}$ subject—satisfied criteria for mild depression (HDI-SF scores between 10 and 12). Emotional control tasks established that the two groups exhibited highly comparable abilities to interpret verbal descriptions of prototypical emotion-inducing situations $[t(40)=$ $-0.80, p=.43]$ and static photographs of emotional faces $[t(40)=-0.59, p=.56]$.

Further background testing revealed that auditory working memory capacity was significantly reduced in the PD group $[t(40)=-3.67, p<.001]$, as was the ability to discriminate nonemotional auditory information according to underlying segmental or suprasegmental (i.e., stress) contrasts [Benton phoneme discrimination, $t(40)=-2.46$, $p=.02$; nonemotional prosody discrimination, $t(40)=$ $-3.36, p=.002]$. Pearson product-moment correlations with Bonferroni-corrected probability levels revealed a strong relationship between working memory scores in the two groups and scores for nonemotional prosody discrimination $(r=0.55, p<.001)$. Performance on the Mattis Dementia Rating Scale was intercorrelated with both working memory $(r=.56, p=.001)$ and nonemotional prosody discrimination $(r=.43 p<.05)$, implying 
that difficulties in working memory may have been central to understanding the PD group's performance in several domains. Scores on the Benton Phoneme Discrimination were not strongly tied to working memory, nonemotional prosody discrimination, or DRS full scores (all $p \mathrm{~s}>.12$ ). To briefly probe whether cognitive or mood differences identified in the PD sample contributed to aberrant decoding of emotional prosody in any of the three conditions, canonical correlations were computed between $Z$ transformed condition scores and independent factors of depression (HDI-SF score), Benton phoneme discrimination (number correct), and a composite score representing auditory processing/working memory abilities (the standardized mean of words recalled in the working memory task and accuracy for nonemotional prosody discrimination, which were highly correlated). The ability of PD subjects to discriminate and to identify prosody was each significantly associated with auditory processing/working memory capabilities (both $r \mathrm{~s}=.51, p \mathrm{~s}<.05$ ). In contrast, the reduced sensitivity of PD patients to target emotion features in the rating condition was not predicted by these independent factors (all $p \mathrm{~s}>.27$ ).

\section{DISCUSSION}

There are accumulating indications that the basal ganglia assume a role in how humans process speech prosody, especially its emotive content (Breitenstein et al., 1998; Cancelliere \& Kertesz, 1990; Karow, Marquardt, \& Marshall, 2001; Kotz et al., 2003; Morris et al., 1999; Pell, 1996; Starkstein et al., 1994). One approach for inferring how the basal ganglia contribute to these (and other) facets of behavior and cognition is to study nondemented adults in the relatively early stages of idiopathic PD, when damage is largely confined to these structures (e.g., SaintCyr, Taylor, \& Lang, 1988). Adopting this approach, we obtained robust new evidence that individuals with PD are less sensitive to prosodic cues about emotion in a novel array of tasks designed to tap this form of information processing; our PD group was impaired in the ability to discriminate, verbally identify, and rate five "basic" attributes of emotionally intoned utterances that were otherwise devoid of meaningful semantic content. Prosodic difficulties emerged in the absence of gross intellectual impairment and were not predicted by the severity of depressive signs in affected individuals with PD (Benke, Bosch, \& Andree, 1998; Breitenstein et al., 2001). Moreover, comprehensive background testing yielded clear signs that deficits for judging the prosodic parameters of utterances were not part of a more generalized impairment in the regulation of emotional stimuli, since PD patients were highly effective at decoding stimuli associated with the five target emotion concepts when they were encountered in other sensory/communicative channels-that is, when they were presented in verbal scenarios (Breitenstein et al., 2001; Pell, 1996; St. Clair, Borod, Sliwinski, Cote, \& Stern, 1998) or as static facial expressions posed by the same actors (Adolphs, Schul, \& Tranel, 1998; Borod et al., 1990; see also Pell \& Leonard, 2003).

In light of these observations, our data support findings from a growing list of research that associates neuropathologic alterations in PD with specific deficits in the decoding of emotional prosody (Blonder et al., 1989; Borod et al., 1990; Breitenstein et al., 1998; Breitenstein et al., 2001; Lloyd, 1999; Pell, 1996; Scott et al., 1984; cf. Caekebeke et al., 1991; Kan et al., 2002). Given the functional importance of interruptions within the striatal complex to the early pathogenesis and clinical presentation of $\mathrm{PD}$, these collective findings imply that understanding emotional prosody is somehow dependent on mechanisms supplied by the intact basal ganglia, which are adversely affected by PD (Blonder et al., 1989; Pell, 1996). Consistent with this point of view, basal ganglia degeneration in Huntington's disease has been separately linked with problems in comprehending basic emotions signaled through prosody (Speedie et al., 1990; Sprengelmeyer et al., 1996). Although neuropathology in both PD and Huntington's disease is known to progressively implicate brain regions outside the basal ganglia (especially cortical sites), the decline in prosodic skills frequently witnessed in these two populations strongly suggests that the basal ganglia provide a substrate for operations involved in emotional prosody recognition (Blonder et al., 1989; Pell, 1996).

The opinion that the basal ganglia serve an important and perhaps mandatory role in the receptive processing of prosody gains further, direct support from neuropsychological investigations of adults with focal basal ganglia pathology and from functional neuroimaging. Cancelliere and Kertesz (1990) assessed emotion comprehension and expression in 46 patients with focal stroke lesions and then applied a CT-overlap technique to associate prosody deficits with lesion site; the authors' overall conclusion was that damage to the basal ganglia corresponded most strongly with detectable impairments in prosody comprehension. Starkstein et al. (1994) also have reported a relationship between (right) basal ganglia lesions and more severe emotional prosody deficits in their survey of 59 consecutive stroke patients, as have other researchers (Bradvik et al., 1991; Karow et al., 2001; Weddell, 1994; cf. Adolphs et al., 2002). Neuroimaging work also speaks to a probable role for the basal ganglia in emotional prosody decoding, bolstering the accumulating evidence from patient group studies. Very recently, Kotz et al. (2003) required 12 listeners to rate emotional prosody from "speechfiltered" (pure prosody) and "normal" speech, using eventrelated functional MR; in both of their conditions, the investigators observed bilateral activation of the putamen, the thalamus, and the head of the caudate, in addition to bilateral cortical activation in temporal and inferior frontal sites. Using PET, Morris et al. (1999) reported activation of the right caudate nucleus, in addition to frontal/temporal cortical regions, in response to emotional vocalizations by 6 male subjects. Although not all the investigations in this small literature have detected basal ganglia activation in 
response to emotional prosody (cf. Buchanan et al., 2000; George et al., 1996; Imaizumi et al., 1997; Mitchell, Elliott, Barry, Cruttenden, \& Woodruff, 2003), the results of Kotz et al.'s well-controlled study were interpreted as a specific call for researchers to explore the important functional relationship between the basal ganglia and emotional speech processing (Kotz et al., 2003).

Given the convergence of many data from PD, Huntington's disease, focal lesion studies, and neuroimaging, one may fairly argue that the basal ganglia are critically involved in certain aspects of emotional prosody decoding. Ourdata from PD imply that the basal ganglia do not contribute uniformly to the mediation of all nonverbal symbols of emotion, since our patients could readily appreciate the significance of emotional faces in identification and rating paradigms similar to those employed here for prosody (Pell \& Leonard, 2003). As we discuss at length in that report, a correspondence between the basal ganglia and the processing of static facial expressions of emotion based on evidence from PD is far less clear-cut than that for emotional prosody. Several investigators have reported that facial expression analysis is impaired in PD (Beatty et al., 1989; Breitenstein et al., 1998; Jacobs, Shuren, Bowers, \& Heilman, 1995), including a recent undertaking that has stressed that these impairments are mitigated to some extent by L-dopa therapy in medicated, rather than unmedicated, adults with the disease (Sprengelmeyer et al., 2003). ${ }^{1}$ However, a comparable number of studies, in addition to ours, have revealed no evidence that emotional face processing is affected by PD (Adolphs et al., 1998; Borod et al., 1990; Dewick, Hanely, Davies, Playfer, \& Turnbull, 1991; Haeske-Dewick, 1996; Pell \& Leonard, 2003; St. Clair et al., 1998). Moreover, in those cases in which emotional prosody and emotional face-processing skills were directly compared, prosodic impairments were often more frequent or pronounced in adults with PD (Blonder et al., 1989; Borod et al., 1990; cf. Kan et al., 2002) or in those with focal basal ganglia lesions (Cancelliere \& Kertesz, 1990; Weddell, 1994). Although more comparative research is clearly needed, the relative lack of consensus in the face-processing literature, coupled with our clear demonstration that only prosody, and not face, processing was disturbed in the present PD sample, encourages researchers to provide an explanation for why interpretive processes more specific to prosody may be disproportionately affected by basal ganglia compromise. A possible mechanism that the basal ganglia may provide to prosody comprehension and supporting rationale is offered below.

An alternative, perhaps additional possibility is that basal ganglia disease is associated with a selective decline in the recognition of particular emotional states independently of the nonverbal channel. In line with the hypothesis that primary human emotions activate distinct neural-processing networks (Adolphs, 2002; Panksepp, 1982), there is a growing literature that links the basal ganglia with the ability to recognize disgust through the face or voice (see Calder, Lawrence, \& Young, 2001, for an overview). The present study allowed emotion-specific effects to be inspected for both identification and rating of happiness, (pleasant) surprise, anger, disgust, and sadness; in the rating condition, our results indicated that PD patients were significantly less sensitive to vocal cues of disgust and sadness, reinforcing previous claims that the basal ganglia may be implicated in the perception of disgust (Gray, Young, Barker, Curtis, \& Gibson, 1997; Sprengelmeyer et al., 1996; Sprengelmeyer et al., 2003; Wang et al., 2003) and perhaps sadness (George et al., 1995; Lane, Reiman, Ahern, Schwartz, \& Davidson, 1997). However, emotion-specific deficits were notably limited to the rating, and not the identification, condition, and the PD patients were unimpaired at recognizing these two emotions from facial expressions (Pell \& Leonard, 2003), implying possible constraints on how the basal ganglia are likely engaged in the regulation of these specific emotions. More research on how the basal ganglia govern the processing of discrete emotional meanings in the prosodic channel, based on a robust inventory of perceptually validated prosodic stimuli such as those presented here, represents a particularly useful direction for extension of these ideas, which at present are based largely on the processing of face stimuli.

The influence of executive resource functions on prosody decoding, especially working memory capacity, which is frequently attenuated in early PD (Gabrieli, Singh, Stebbins, \& Goetz, 1996; Owen et al., 1992), was also key to understanding the implications of our prosody measures. Some researchers have emphasized the dependence of receptive prosodic deficits on more primary executive resource/working memory limitations in PD (Benke et al., 1998; Breitenstein et al., 2001). Prosody decoding, although not fully understood, is undeniably supported by operations that permit sustained monitoring and analysis of spectrotemporal changes of a dynamic auditory stimulus held in temporary storage (Pihan, Altenmüller, Hertrich, \& Ackermann, 2000; Rama et al., 2001). These operations are frequently exacerbated by conventional off-line tests of prosody comprehension (Breitenstein et al., 2001), a pattern that may obscure whether prosodic failures are due to a breakdown in frontal-striatal structures implicated in working memory (Braver et al., 1997; Rypma, Prabhakaran, Desmond, Glover, \& Gabrieli, 1999) or to a more direct contribution of basal ganglia sectors to processing the emotional value of prosodic cues.

Standardized testing of the present PD patients revealed no substantive difficulties in the regulation of attention or the shifting of mental sets using common frontal lobe tasks (Trail-making test, WCST), although a marked decline in auditory discrimination and auditory-verbal working memory (listening span) was confirmed in this group. Critically, an important relationship was documented between a composite measure of nonemotional auditory processing abilities and performance in two of the three emotional prosody conditions: discrimination and identification. These data reaffirm that working memory restrictions are a significant predictor of emotional prosody comprehension when subjects are required to categorize the intended verbal meaning of prosody in the ubiquitous forced-choice response paradigm (Breitenstein et al., 
2001). However, deficits in working memory in our PD patients did not account for their aberrant recognition of emotional prosody in the rating condition, which was included to mitigate executive task demands on processes of prosody interpretation and response generation for the same stimuli. Thus, the results of this broader survey of emotional prosody decoding in PD patients emphasize that alterations in fronto-striatal functioning/working memory are critical to the understanding of performance in certain tasks but that they do not explain the full origins of these prosodic impairments. Rather, as was argued above, prosodic abnormalities in early PD and other forms of basal ganglia disease are likely to reflect distinct functional properties of the basal ganglia that participate directly in certain aspects of prosody decoding (Kotz et al., 2003).

\section{A Role for the Basal Ganglia in Prosodic Processing}

There is a growing appreciation that cortical and subcortical structures engage in fundamental and distinct aspects of information processing during cognitive tasks concerned with emotion, memory, and learning (Adolphs, 2002; Heilman, 1997; LeDoux, 1993). Although our data signal the importance of looking at how the basal ganglia support prosodic functions within distributed brain networks devoted to these skills (Adolphs et al., 2002; Baum $\&$ Pell, 1999), relevant data are still limited and preclude a detailed interpretation of how discrete prosodic functions may be linked to the basal ganglia or an extensive commentary on localization of prosodic functions within the basal ganglia. Nonetheless, critical insights may be drawn from broader descriptions of the cognitive capacity of the basal ganglia that will lend coherence to the emotional prosody literature, advancing future perspectives of the cognitive neurology of these abilities.

Long-standing research characterizing the motor functions of the basal ganglia has suggested that basal ganglia activity is initiated at cortical levels and that striatal nuclei serve to modify the transmission of cortically generated information via motor cortico-striatal-thalamocortical loops (Alexander, DeLong, \& Strick, 1986). The nature of basal ganglia activity within the "complex" prefrontal loops involved in cognitive functions is somewhat less understood, although striatal zones, such as the caudate, have been ascribed an analogous role in modulating higher cortical processes for nonmotor sensory information (Afifi, 1994; Bhatia \& Marsden, 1994; Meck \& Benson, 2002; Rinne et al., 2000; Wise, Murray, \& Gerfen, 1996). These researchers have shown that the striatum possesses a capacity to integrate or potentiate aspects of the cortically initiated dataflow for elaboration by cortical (especially prefrontal) afferents of striatal-pallidothalamocortical pathways; these striatal influences on information processing represent direct contributions of the basal ganglia dissociable from those of fronto-striatal connections or other functionally integrated structures that may act on the data (Hay, Moscovitch, \& Levine, 2002; Knowlton, 1996; Meck \& Benson, 2002; Owen et al., 1993; Poldrack, Prabhakaran, Seger, \& Gabrieli, 1999).
There are important reasons to suspect that the basal ganglia (striatum) fulfill a similar role in the decoding of emotional and, perhaps, other attributes of speech prosody, lending coherence to these signals during the comprehension process. Anatomically, the striatum is well positioned to assume a potentiating or reinforcing role in emotional prosody decoding; striatal nuclei receive major inputs from prefrontal areas, as well as multiple projections from functionally related sites throughout the cortex, the thalamus, and the limbic system (amygdala; Afifi, 1994). This dense connectivity with structures involved in cognition, attention, and affective functioning places the striatum in an arguably privileged location to act on input from a variety of sites, from limbic and nonlimbic sources, permitting access to the diverse range of information presumably required to evaluate the significance of emotional stimuli and events (Adolphs, 2002; LeDoux, 1989; Rolls, 1990). Although it is unclear exactly how a basal ganglia mechanism devoted to the mediation of prosodic features of the human voice would operate, the striatum is known to act on incoming sensory information to reinforce the significance of these events, facilitating a contextually appropriate behavioral response at the level of the neocortex (Lieberman, 2000; Meck \& Benson, 2002). One can reasonably speculate that this filtering function of the basal ganglia, if applied to an incoming spectrotemporal stimulus, could play a critical role in facilitating cortical responses about the value of stimulus features detected through efficient mapping of prosodic features onto higher order representations (Pell, 1996).

Justification for why basal ganglia functioning may be critical to prosody refers to the well-recognized role of the striatum in timing behavior, including receptive operations that act on temporal sensory events. Clinical and neuroimaging evidence has implicated fronto-striatal circuitry in the nonmotor operations that govern time perception, the encoding of time intervals, and the temporal discrimination and resolution of sensory events (Artieda, Pastor, Lacruz, \& Obeso, 1992; Harrington et al., 1998; Meck \& Benson, 2002; Rao, Mayer, \& Harrington, 2001). These time-dependent processes, like those for motor timing, are critically reliant on the striatum (Artieda et al., 1992; Harrington et al., 1998). It has thus been proposed that one of the functional advantages afforded by the basal ganglia during information processing is to enable organisms to adapt to changing temporal contexts, or to sensory events of an inherently temporal nature, by reinforcing attributes of the cue sequences of greatest behavioral significance (Lieberman, 2000; Meck \& Benson, 2002). The perceived socio-emotive significance of prosodic stimuli evolves from a temporal string of affective cue sequences, predominantly global pitch alterations over an extended time window (Ladd, Silverman, Tolkmitt, Bergmann, \& Scherer, 1985; Pell, 2001). This behavioral task is likely to benefit greatly from a basal ganglia mechanism attuned to predicting the value of cue sequences within temporal sensory events (see Lieberman, 2000, for a critical discussion of this view). The idea that basal ganglia participation is triggered by the temporal constitution of prosodic dis- 
plays has gained some direct support in the prosody literature through a recent investigation in which PD patients were abnormally sensitive to temporal modifications of emotional stimuli presented for perceptual recognition (Breitenstein et al., 2001).

Pending further data, one can hypothesize that loss of striatal dopamine in early PD culminates in "noisy" output within striato-pallido-thalamo-cortical pathways (e.g., Bhatia \& Marsden, 1994), yielding a critical reduction or withdrawal of a striatally based mechanism that lends coherence to spectrotemporal patterns in speech (Lieberman, 2000). These faulty outputs associated with basal ganglia disease manifest as reduced sensitivity to the meaning encoded by prosodic cue sequences, owing to the remote influences of this aberrant mechanism on functionally interconnected areas of the cortex where higher order interpretative processes are accomplished, particularly the (right) inferior prefrontal cortex (Adolphs et al., 2002; Buchanan et al., 2000; George et al., 1996; Kotz et al., 2003). Alternatively, the role of the basal ganglia in emotional prosody recognition may more accurately reflect the known motor functions of these structures; if one accepts the view that comprehension of emotional stimuli is accomplished by reconstructing or simulating the somatic/ motor components associated with the production of emotional states, it is possible that the basal ganglia are recruited in concert with other motor-related brain regions to derive knowledge about emotion displays (Adolphs et al., 2002).

Future research is called for to test these claims, leading to more sophisticated accounts of how the basal ganglia contribute to emotional prosody and of the functional neuroanatomy of these processes within the basal ganglia. Determining the functional relationship between the basal ganglia, the amygdala, and the relative dominance of rightsided cortical regions (e.g., Pell, 1998; Pihan et al., 2000) at various stages of emotional prosody appraisal and conscious interpretation of these events will prove especially insightful. Finally, in advancing the idea that the basal ganglia are attuned to the representational value of prosodic cue sequences, the specificity of this mechanism to only emotional aspects of prosody is still suspect and will require controlled study; fine-grained analysis of prosodic processing in emotional versus linguistic contexts was beyond the purview of this report, allowing for the possibility that prosodic deficits in PD are highly pronounced but not limited to emotional-processing contexts, as is implied somewhat by our data and elsewhere in the prosody literature (Blonder et al., 1989; Lloyd, 1999; Pell, 1996).

\section{REFERENCES}

ADOLPHS, R. (2002). Neural systems for recognizing emotion. Current Opinion in Neurobiology, 12, 169-177.

AdOLPHS, R., DAmasio, H., \& TRANEL, D. (2002). Neural systems for recognition of emotional prosody: A 3-D lesion study. Emotion, 2, 23-51.

ADOLPHS, R., SCHUL, R., \& TRANEL, D. (1998). Intact recognition of facial emotion in Parkinson's disease. Neuropsychology, 12, 253-258.

ADOLPHS, R., \& TRANEL, D. (1999). Intact recognition of emotional prosody following amygdala damage. Neuropsychologia, 37, 1285-1292.
Adolphs, R., Tranel, D., Damasio, H., \& Damasio, A. (1995). Fear and the human amygdala. Journal of Neuroscience, 15, 5879-5892.

AFIFI, A. K. (1994). Basal ganglia: Functional anatomy and physiology: Pt. 2. Journal of Child Neurology, 9, 352-361.

Alexander, G., DeLong, M., \& Strick, P. (1986). Parallel organization of functionally segregated circuits linking basal ganglia and cortex. Annual Review of Neuroscience, 9, 337-381.

Artieda, J., PAstor, M., LACruZ, F., \& Obeso, J. (1992). Temporal discrimination is abnormal in Parkinson's disease. Brain, 115, 199-210.

BAUM, S., \& PELL, M. (1999). The neural bases of prosody: Insights from lesion studies and neuroimaging. Aphasiology, 13, 581-608.

Beatty, W. W., Goodkin, D. E., Weir, W. S., Staton, R. D., MonSON, N., \& BEATTY,P. A. (1989). Affective judgments by patients with Parkinson's disease or chronic prog ressive multiple sclerosis. Bulletin of the Psychonomic Society, 27, 361-364.

Benke, T., Bosch, S., \& ANDREe, B. (1998). A study of emotional processing in Parkinson's disease. Brain \& Cognition, 38, 36-52.

Bhatia, K. P., \& MARsden, C. D. (1994). The behavioural and motor consequences of focal lesions of the basal ganglia in man. Brain, 117, 859-876.

BLONDER,L. X., GUR, R. E., \& GUR, R. C. (1989). The effects of right and left hemiparkinsonism on prosody. Brain \& Language, 36, 193-207.

BoroD, J. C. (1993). Cerebral mechanisms underlying facial, prosodic, and lexical emotional expression: A review of neuropsychological studies and methodological issues. Neuropsychology, 7, 445-463.

Borod, J. C., Welkowitz, J., Alpert, M., Brozgold, A. Z., Martin, C., Peselow, E., \& Diller, L. (1990). Parameters of emotional processing in neuropsychiatric disorders: Conceptual issues and a battery of tests. Journal of Communication Disorders, 23, 247-271.

Bradvik, B., Dravins, C., Holtas, S., Rosen, I., Ryding, E., \& INGVAR, D. H. (1991). Disturbances of speech prosody following right hemisphere infarcts. Acta Neurologica Scandanivica, 54, 114-126.

Braver, T., Cohen, J., Nystrom, L., Jonides, J., Smith, E., \& Noll, D. (1997). A parametric study of prefrontal cortex involvement in human working memory. Neurolmage, 5, 49-62.

Breitenste In, C., DAUM, I., \& ACKermann, H. (1998). Emotional processing following cortical and subcortical brain damage: Contribution of the fronto-striatal circuitry. Behavioural Neurology, 11, 29-42.

Breitenste in, C., VAn Lancker, D., Daum, I., \& Waters, C. (2001). Impaired perception of vocal emotions in Parkinson's disease: Influence of speech time processing and executive functioning. Brain \& Cognition, 45, 277-314.

Buchanan, T. W., Lutz, K., Mirzazade, S., SPecht, K., Jon Shah, N., ZILLES, K., \& JANCKE, L. (2000). Recognition of emotional prosody and verbal components of spoken language: An fMRI study. Cognitive Brain Research, 9, 227-238.

CAE KEBEKE, J. F. V., JeNNE KENS-SChinKEL, A., VAN DER LindEN, M. E., Buruma, O. J. S., \& Roos, R. A. C. (1991). The interpretation of dysprosody in patients with Parkinson's disease. Journal of Neurology, Neurosurgery, \& Psychiatry, 54, 145-148.

Calder, A., Keane, F., Manes, F., Antoun, N., \& Young, A. (2000). Impaired recognition and experience of disgust following brain injury. Nature Neuroscience, 3, 1077-1078.

CAlder, A., Lawrence, A., \& Young, A. (2001). Neuropsychology of fear and loathing. Nature Reviews Neuroscience, 2, 352-363.

CAlne, D., Snow, B., \& LeE, C. (1992). Criteria for diagnosing Parkinson's disease. Annals of Neurology, 32, S125-S127.

CANCelliere, A. E. B., \& Kertesz, A. (1990). Lesion localization in acquired deficits of emotional expression and comprehension. Brain \& Cognition, 13, 133-147.

Dewick, H. C., Hanley, J. R., Davies, A. D., Playfer, J., \& Turnbull, C. (1991). Perception and memory for faces in Parkinson's disease. $\mathrm{Neu}$ ropsychologia, 29, 785-802.

Freeman, A., Ciliax, B., Bakay, R., Daley, J., Miller, R. D., KeatING, G., LEVEY, A., \& RYE, D. (2001). Nigrostriatal collaterals to thalamus degenerate in Parkinsonian animal models. Annals of Neurology, 50, 321-329.

Gabrieli, J. D. E., Singh, J., Stebbins, G. T., \& Goetz, C. G. (1996). Reduced working memory span in Parkinson's Disease: Evidence for the role of a frontostriatal system in working and strategic memory. Neuropsychology, 10, 322-332. 
Gandour, J., Wong, D., \& Hutchins, G. (1998). Pitch processing in the human brain is influenced by language experience. NeuroReport, 9, 2115-2119.

George, M. S., Ketter, T. A., Pare Kh, P. I., Horowitz, B., HerSCOVITCH, P., \& POST, R. (1995). Brain activity during transient sadness and happiness in healthy women. American Journal of Psychiatry, 152, 341-351.

George, M. S., Parekh, P. I., Rosinsky, N., Ketter, T. A., Kimbrell, T. A., Heilman, K. M., Herscovitch, P., \& Post, R. M. (1996). Understanding emotional prosody activates right hemisphere regions. Archives of Neurology, 53, 665-670.

Gray, J. M., Young, A. W., Barker, W. A., Curtis, A., \& Gibson, D. (1997). Impaired recognition of disgust in Huntington's disease gene carriers. Brain, 120, 2029-2038.

HAESKE-DEWICK, H. (1996). Are perception and memory for faces influenced by a specific age at onset factor in Parkinson's disease? Neuropsychologia, 34, 315-320.

Harrington, D., HaAland, K., \& Hermanowicz, N. (1998). Temporal processing in the basal ganglia. Neuropsychology, 12, 3-12.

Hay, J., Moscovitch, M., \& LeVINE, B. (2002). Dissociating habit and recollection: Evidence from Parkinson's disease, amnesia and focal lesion patients. Neuropsychologia, 40, 1324-1334.

HeILMAN, K. M. (1997). The neurobiology of emotional experience. Journal of Neuropsychiatry \& Clinical Neurosciences, 9, 439-448.

Imaizumi, S., Mori, K., Kiritani, S., KaWAshima, R., Sugiura, M., Fukuda, H., Itoh, K., Kato, T., Nakamura, A., Hatano, K., KoJIMA, S., \& NAKAMURA, K. (1997). Vocal identification of speaker and emotion activates different brain regions. NeuroReport, 8, 2809-2812.

JACOBS, D., SHuREN, J., Bowers, D., \& HeIlmAN, K. (1995). Emotional facial imagery, perception, and expression in Parkinson's disease. Neurology, 45, 1696-1702.

Kan, Y., Kawamura, M., Hasegawa, Y., Mochizuki, S., \& NaKaMURA, K. (2002). Recognition of emotion from facial, prosodic and written verbal stimuli in Parkinson's disease. Cortex, 38, 623-630.

Karow, C., Marquardt, T., \& Marshall, R. (2001). Affective processing in left and right hemisphere brain-damaged subjects with and without subcortical involvement. Aphasiology, 15, 715-729.

KNOWLton, B. J. (1996). A neostriatal habit learning system in humans. Science, 273, 1399-1402.

Kotz, S., Meyer, M., Alter, K., Besson, M., von Cramon, Y., \& FRIEDERICI, A. (2003). On the lateralization of emotional prosody: An event-related functional MR investigation. Brain \& Language, 86, 366-376.

Ladd, D. R., Silverman, K. E. A., Tolkmitt, F., Bergmann, G., \& SCHERER, K. R. (1985). Evidence for the independent function of intonation contour type, voice quality, and $\mathrm{F} 0$ range in signaling speaker affect. Journal of the Acoustical Society of America, 78, 435-444.

Lane, R. D., Reiman, E. M., Ahern, G. L., Schwartz, G. E., \& DAVIDSON, R. J. (1997). Neuroanatomical correlates of happiness, sadness, and disgust. American Journal of Psychiatry, 154, 926-933.

LEDoux, J. E. (1989). Cognitive-emotional interactions in the brain. Cognition \& Emotion, 3, 267-289.

LEDoux, J. E. (1993). Emotional memory systems in the brain. Behavioural Brain Research, 58, 69-79.

LIEBERMAN, M. D. (2000). Intuition: A social cognitive neuroscience approach. Psychological Bulletin, 126, 109-137.

LloYd, A. J. (1999). Comprehension of prosody in Parkinson's disease. Cortex, 35, 389-402.

MECK, W., \& BENSON, A. (2002). Dissecting the brain's internal clock: How frontal-striatal circuitry keeps time and shifts attention. Brain \& Cognition, 48, 195-211.

Mitchell, R., Elliott, R., Barry, M., Cruttenden, A., \& WoodRUFF, P. (2003). The neural response to emotional prosody, as revealed by functional magnetic resonance imaging. Neuropsychologia, 41, 1410-1421.

Morris, J. S., ScotT, S. K., \& Dolan, R. J. (1999). Saying it with feeling: Neural responses to emotional vocalizations. Neuropsychologia, 37, 1155-1163.

Owen, A. M., James, M., Leigh, P. N., Summers, B. A., Marsden, C. D., Quinn, N. P., LANGe, K. W., \& Robbins, T. W. (1992). Fronto- striatal cognitive deficits at different stages of Parkinson's disease. Brain, 115, 1727-1751.

Owen, A. M., Roberts, A. C., Hodges, J. R., Summers, B. A., Polkey, C. E., \& RobBins, T. W. (1993). Contrasting mechanisms of impaired attentional set-shifting in patients with frontal lobe damage or Parkinson's disease. Brain, 116, 1159-1175.

PANKSE PP, J. (1982). Toward a general psychobiological theory of emotions. Behavioral \& Brain Sciences, 5, 407-467.

Pell, M. D. (1996). On the receptive prosodic loss in Parkinson's disease. Cortex, 32, 693-704.

PELl, M. D. (1998). Recognition of prosody following unilateral brain lesion: Influence of functional and structural attributes of prosodic contours. Neuropsychologia, 36, 701-715.

PELL, M. D. (2001). Influence of emotion and focus location on prosody in matched statements and questions. Journal of the Acoustical Society of America, 109, 1668-1680.

PELL, M. D. (2002). Evaluation of nonverbal emotion in face and voice: Some preliminary findings on a new battery of tests. Brain \& Cognition, 48, 499-504.

PELL, M. D., \& BAUM, S. R. (1997). The ability to perceive and comprehend intonation in linguistic and affective contexts by brain-damaged adults. Brain \& Language, 57, 80-99.

PELL, M. D., \& LEONARD, C. (2003). Emotional face processing in early Parkinson's disease. Manuscript submitted for publication.

Pihan, H., Altenmüller, E., \& ACKermann, H. (1997). The cortical processing of perceived emotion: A DC-potential study on affective speech prosody. NeuroReport, 8, 623-627.

Pihan, H., Altenmüller, E., Hertrich, I., \& ACKermann, H. (2000). Cortical activation patterns of affective speech processing depend on concurrent demands on the subvocal rehearsal system: A DC-potential study. Brain, 123, 2338-2349.

Poldrack, R. A., Prabhakaran, V., Seger, C. A., \& Gabrieli, J. D. E. (1999). Striatal activation during acquisition of a cognitive skill. Neuropsychology, 13, 564-574.

RAMa, P., MARTinkauppi, S., Linnankoski, I., Koivisto, J., Aronen, H., \& CARLSON, S. (2001). Working memory of identification of emotional vocal expressions: An fMRI study. NeuroImage, 13, 1090-1101.

RAO, S., MAYER,A., \& HARrINGTON,D. (2001). The evolution of brain activation during temporal processing. Nature Neuroscience, 4, 317-323.

Rinne, J. O., Portin, R., Ruottinen, H., Nurmi, E., Bergman, J., HaAparanta, M., \& Solin, O. (2000). Cognitive impairment and the brain dopaminergic system in Parkinson disease. Archives of Neurology, 57, 470-475.

Rolls, E. T. (1990). A theory of emotion, and its application to understanding the neural basis of emotion. Cognition \& Emotion, 4, 161-190.

Ross, E. D., Thompson, R. D., \& Yenkosky, J. (1997). Lateralization of affective prosody in brain and the collosal integration of hemispheric language functions. Brain \& Language, 56, 27-54.

Rypma, B., Prabhakaran, V., Desmond, J., Glover, G., \& Gabrie Li, J. (1999). Load-dependent roles of frontal brain regions in the maintenance of working memory. NeuroImage, 2, 216-226.

SAint-Cyr, J. A., TAYlor, A. E., \& LANG, A. E. (1988). Procedural learning and neostriatal dysfunction in man. Brain, 111, 941-959.

SCHERER, K. R. (1993). Neuroscience projections to current debates in emotion psychology. Cognition \& Emotion, 7, 1-41.

Scherer, K. R., BANSE, R., \& WAllbott, H. (2001). Emotion inferences from vocal expression correlate across languages and cultures. Journal of Cross-Cultural Psychology, 32, 76-92.

SCOTT, S., CAIRD, F., \& Williams, B. (1984). Evidence for an apparent sensory speech disorder in Parkinson's disease. Journal of Neurology, Neurosurgery, and Psychiatry, 47, 840-843.

Speedie, L. J., Brake, N., Folstein, S. E., Bowers, D., \& Heilman, K. E. (1990). Comprehension of prosody in Huntington's disease. Journal of Neurology, Neurosurgery, and Psychiatry, 53, 607-610. Sprengelmeyer, R., Young, A. W., Calder, A. J., Karnat, A., Lange, H., Homberg, V., Perrett, D. I., \& Rowland, D. (1996). Loss of disgust: Perception of faces and emotions in Huntington's disease. Brain, 119, 1647-1665.

Sprengelmeyer, R., Young, A. W., Mahn, K., Schroeder, U., Woitalla, D., Büttner, T., Kuhn, W., \& Przuntek, H. (2003). Fa- 
cial expression recognition in people with medicated and unmedicated Parkinson's disease. Neuropsychologia, 41, 1047-1057.

Starkstein, S. E., Federoff, J. P., Price, T. R., Leiguarda, R. C., \& RoBINSON, R. G. (1994). Neuropsychological and neuroradiologic correlates of emotional prosody comprehension. Neurology, 44, 515-522.

St. Clair, J., Borod, J. C., Sliwinski, M., Cote, L. J., \& Stern, Y. (1998). Cognitive and affective functioning in Parkinson's disease patients with lateralized motor signs. Journal of Clinical \& Experimental Neuropsychology, 20, 320-327.

Tompkins, C. A., Bloise, C. G. R., Timko, M. L., \& Baumgaertner, A. (1994). Working memory and inference revision in brain-damaged and normally aging adults. Journal of Speech \& Hearing Research, 37, 896-912.

WanG, K., Hoosain, R., YANG, R.-M., MenG, Y., \& WANG, C.-Q. (2003). Impairment of recognition of disgust in Chinese with Huntington's or Wilson's disease. Neuropsychologia, 41, 527-537.
WEDDELL, R. (1994). Effects of subcortical lesion site on human emotional behavior. Brain \& Cognition, 25, 161-193.

Wise, S., MurRay, E., \& GerFen, C. (1996). The frontal cortex-basal ganglia system in primates. Critical Reviews in Neurobiology, 10, 317-356.

\section{NOTE}

1. All remaining investigations of emotional face and emotional prosody processing in PD patients are derived strictly from medicated patients, precluding an analysis of this variable within the broader literature at this time.

(Manuscript received April 8, 2003; revision accepted for publication October 1, 2003.) 